\section{Unilateral sudden hearing loss due to otosyphilis} Jeroen B. van Rijswijk ${ }^{3}$

'Department of Otorhinolaryngology, Head \& Neck Surgery, Erasmus Medical Center Rotterdam, Rotterdam;

${ }^{2}$ Department of Neurology, Admiral de Ruyter Hospital, Goes; ${ }^{3}$ Department of Otorhinolaryngology, Head \& Neck Surgery and Facial Plastics, Admiral de Ruyter Hospital, Goes, The Netherlands

\section{Abstract}

The differential diagnosis of sudden hearing loss is very extensive. However, in most patients, no underlying pathology will be found and the patient is consequently diagnosed with idiopathic sudden hearing loss. Nevertheless, it is important that one stays alert in everyday routine practice to less common but potentially treatable pathology. We report a case of a 53year old patient with sudden unilateral hearing loss and tinnitus caused by otosyphilis. Diagnosis, therapy and the incidence of (oto)syphilis are discussed. Also, as ENT-surgeons, it is important to realise that syphilis is still with us, and that its prevalence might well increase in the near future.

\section{Introduction}

The differential diagnosis of sudden hearing loss is very extensive including among others acoustic neuroma, vascular alterations, immune-mediated disorders, Meniere's disease, membrane rupture and infectious diseases, viral or bacterial in origin. However, in most patients, no underlying pathology will be found, even with the tests available today, and the patient is consequently diagnosed with idiopathic sudden hearing loss. Nevertheless, it is important that one stays alert in everyday routine practice to less common but potentially treatable pathology. One of these potentially treatable pathologies is (oto)syphilis, which before the development of penicillin used to be a very common disease. The hope of eradication, however, was unrealistic and over the last ten years the incidence of syphilis has increased again, particularly in Eastern Europe and Russia, but also in the homo- and bisexual communities of large cities in Western Europe. ${ }^{1,2}$

\section{Case Report}

A 53-year old unmarried male presented with a 2-week, sudden unset history of vertigo, tinnitus and sudden hearing loss of the right ear. He also reported headaches over a longer time period and night sweats for nine months; generalpractioners chest X-ray was negative. The general ENT examinations were normal, except for the Weber's tuning fork test lateralizing to the left. The initial tone and speech audiogram revealed total deafness on the right side and a combined hearing loss of $30 \mathrm{~dB}$ on the left side (Figure 1) with $100 \%$ speech discrimination. Other neurological tests and magnetic resonance imaging (MRI) were normal. Despite the fact that the duration of the complaints was longer than one week, the patient expressed a marked preference for a prednisolone oral treatment, which was subsequently prescribed to him, unfortunately without any subjective improvement. After the initial laboratory work up had showed a positive Treponema Pallidum Haemagglutination Assay (TPHA) of more than 20480 and a Rapid Plasma Reagin (RPR) test of 32 units, a more detailed investigation by our
Correspondence: Jeroen B. van Rijswijk, Department of Otorhinolaryngology, Head \& Neck Surgery and Facial Plastics, Admiral de Ruyter Hospital, Goes, Westzeedijk 230 - 3016 AN Rotterdam, The Netherlands.

E-mail: jeroen@vanryswyk.nl

Key words: syphilis, otosyphilis, neurosyphilis, sensorineural hearing loss.

Received for publication: 28 September 2011. Revision received: 28 September 2011. Accepted for publication: 6 November 2011 .

This work is licensed under a Creative Commons Attribution NonCommercial 3.0 License (CC BYNC 3.0).

(C) Copyright M.J. de Goffau et al., 2011

Licensee PAGEPress, Italy

Clinics and Practice 2011; 1:e133

doi:10.4081/cp.2011.e133

neurology department also revealed a short period of penis-ulceration, enlargements of the inguinal glands and perianal pain for three months. An autoimmune profile, Borrelia and
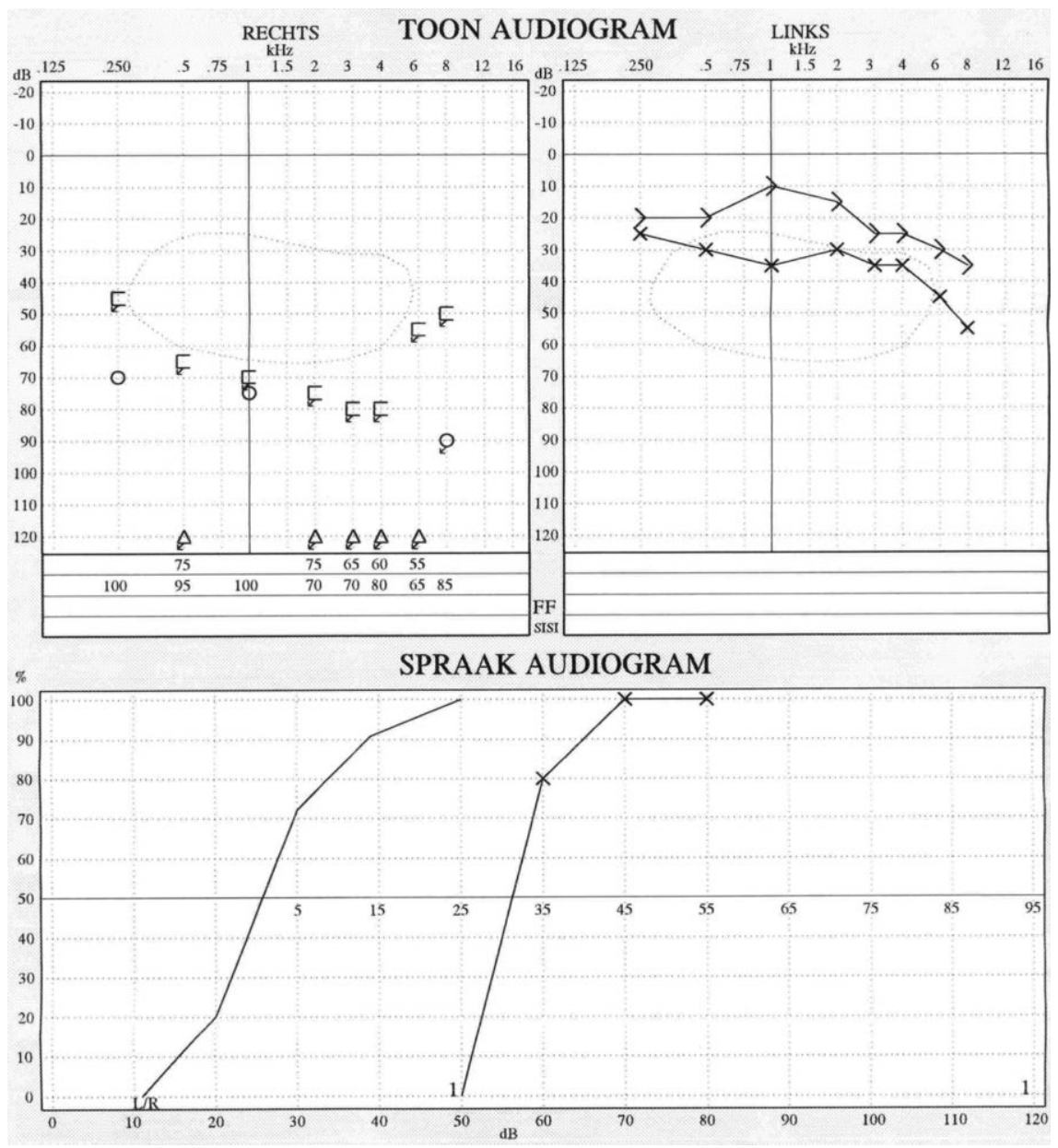

Figure 1. Tone and speech audiogram at initial presentation. 
HIV serology were all-negative. A lumbar puncture performed before starting the treatment showed increased cerebrospinalfluid (CSF) protein, leukocytosis, a TPHA of 2048 and a RPR test of 1 unit. With respect to the CSF abnormalities, the patient was diagnosed with and treated as having neurosyphilis with 2 gram of ceftriaxon intravenously per day for two weeks. Repeated tone and speech audiograms three and six months after therapy showed a minimal improvement for tone perception on the right side, without any speech discrimination and an unchanged sensorineuronal hearing loss on the left side (Figure 2). Unfortunately, the severe disturbing right-sided tinnitus did not improve after therapy but became tolerable with tinnitus rehabilitation/counseling by our local audiology center. Mild imbalance persisted for which no additional therapy or measures were necessary.

\section{Discussion}

Syphilis is a chronic, multi-organ, sexually transmitted infection caused by the anerobic filamentous spirochete Treponoma pallidum. It is often called the Great Imitator because syphilis symptoms resemble those of other common diseases. Otosyphilis is well known as a complication of syphilis. Otosyphilis is usually diagnosed by various cochleo-vestibular symptoms, a positive serological test (TPHA, RPR and or VDRL) and the exclusion of other causes. Accompanied sensineuronal hearing loss may be unilateral or bilateral, usually progresses rapidly and may have a sudden onset as in our case. ${ }^{3}$ Otosyphilis can occur both in congenital and acquired syphilis. In the latter, hearing loss may occur in early or late stages of the disease. ${ }^{4}$ Recommended antibiotic treatment is the same as for neurosyphilis. ${ }^{5}$ Intravenous penicillin is the recommended treatment with ceftriaxone also being effective when used in multiple-dose regimens. ${ }^{5,6}$ In addition to intravenous antibiotics, corticosteroids are usually administered in otosyphilis, probably improving the chances for a favorable treatment hearing outcome ${ }^{7}$ plus preventing a possible Jarisch-Herxheimer reaction.

The little data that exist on the treatment of early disease suggests complete recovery may
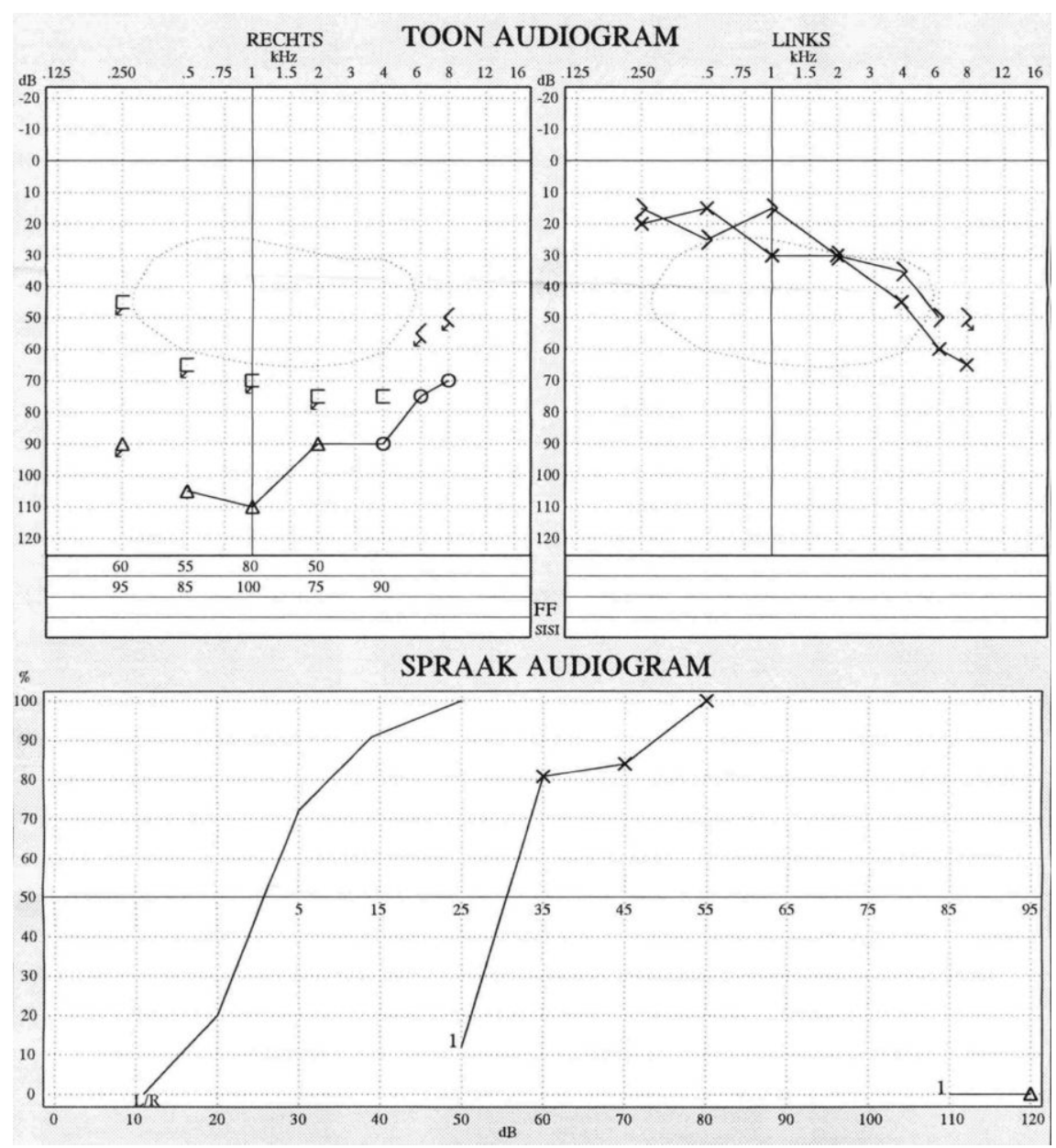

Figure 2. Tone and speech audiogram three months after therapy.

occur if treatment is commenced within weeks of symptom onset. ${ }^{3,7}$ Younger patients might have a better prognosis and patients with unilateral hearing loss might have better response rates than patients with bilateral loss. Also, fluctuation in hearing prior to treatment improves the chances of a persistent hearing improvement after therapy. Outcome is generally poor when symptoms have been present for longer periods, or if hearing loss is absolute prior to treatment, as in our case. ${ }^{7}$

Over the last decades of the twentieth century, the incidence of syphilis continued to fall reaching an all time low in the US in 2000 , with 2.1 cases per 100,000 persons. ${ }^{8}$ Thereafter, over the last ten years, a significant resurgence of this disease has been reported in several countries such as the USA, Canada, England, France, Spain, Ireland, Eastern Europe, Russia and China, with syphilis rates in the USA being approximately similar to European levels. ${ }^{9}$ Main epidemiology changes, at the basis of increasing prevalence, reflect the sex industry, sexual promiscuity, the decrease in use of barrier protection (i.e. condoms) due to a false sense of security that today sexually transmitted diseases are curable and to a lack of pertinent knowledge. Today, more than $50-60 \%$ of new cases of syphilis occur in men who have sex with men and are strongly associated with HIV coinfection and high-risk sexual behavior. ${ }^{2,10}$

\section{Conclusions}

Syphilis is still with us and its prevalence might well further increase in the near future. Otosyphilis is a rare complication of syphilis and although seldom, it is one of the few sudden hearing loss conditions that is amenable to treatment. It should always be taken into consideration in patients suffering from sudden uni- or bilateral sensorineuroal, fluctuating hearing loss, tinnitus and or vertigo. If diagnosed and treated early, hearing loss may be reversible. Treatment might also prevent further hearing loss and / or other late sequela of syphilis (cardiovascular, neurological-psychiatric, ophthalmological, etc.).

\section{References}

1. Klemm E, Wollinga U. Otosyphilis: report on six cases. J Eur Acad Dermatol Venereol 2004;18:429-34.

2. Song JJ, Lee HM, Chae SW, Hwang SJ. Bilateral otosyphilis in a patient with HIV infection. Eur Arch Otorhinolaryngol 2005; 262:972-4.

3. Vercoe GS. The effect of early syphilis on 
the inner ear and auditory nerves. $\mathrm{J}$ Laryngol Otol 1976;90:853-61.

4. Rodgers CA, Murphy S. Diagnosis of neurosyphilis: appraisal of clinical caseload. Genitourin Med 1997;73:528-32.

5. Kingston M, French P, Goh B, et al. UK National Guidelines on the Management of Syphilis 2008. Int J STD AIDS 2008;19: 729-40.
6. Stoner BP. Current controversies in the management of adult syphilis. Clin Infect Dis 2007;44:S130-46.

7. Gleich LL, Linstrom CJ, Kimmelman CP. Otosyphilis: a diagnostic and therapeutic dilemma. Laryngoscope 1992;102:1255-9.

8. Primary and secondary syphilis - United States, 2000-2001. MMWR Morb Mortal Wkly Rep 2002;51:971-3.
9. Ficarra G, Carlos R. Syphilis: The Renaissance of an Old Disease with Oral Implications. Head and Neck Pathol 2009;3:195206.

10. Kent ME, Romanelli F. Reexamining syphilis: an update on epidemiology, clinical manifestations, and management. Ann Pharmacother 2008;42:226-36. 